\title{
Murine gammaherpesvirus 68 glycoprotein 150 does not contribute to latency amplification in vivo
}

Romana Ruiss ${ }^{1 \dagger}$, Shinji Ohno ${ }^{2,4 \dagger}$, Beatrix Steer ${ }^{2}$, Reinhard Zeidler ${ }^{3}$ and Heiko Adler ${ }^{2 *}$

\begin{abstract}
Background: Murine gammaherpesvirus 68 (MHV-68) is used as a model to study the function of gammaherpesvirus glycoproteins. gp150 of MHV-68, encoded by open reading frame M7, is a positional homolog of gp350/220 of EBV and of gp35/37 of KSHV. Since it had been proposed that gp350/220 of EBV might be a suitable vaccine antigen to protect from EBV-associated diseases, gp150 has been applied as a model vaccine in the MHV-68 system. When analyzing the function of gp150, previous studies yielded conflicting results on the role of gp150 in latency amplification, and disparities between the mutant viruses which had been analyzed were blamed for the observed differences.

Results: To further develop MHV-68 as model to study the function of gammaherpesvirus glycoproteins in vivo, it is important to know whether gp150 contributes to latency amplification or not. Thus, we re-evaluated this question by testing a number of gp150 mutants side by side. Our results suggest that gp150 is dispensable for latency amplification. Furthermore, we investigated the effect of vaccination with gp150 using gp150-containing exosomes. Vaccination with gp150 induced a strong humoral and cellular immune response, yet it did not affect a subsequent MHV-68 challenge infection.
\end{abstract}

Conclusions: In this study, we found no evidence for a role of gp150 in latency amplification. The previously observed contradictory results on the role of gp150 in latency amplification were not related to differences between the mutant viruses which had been used.

Keywords: MHV-68, gp150, Latency amplification, Vaccination, Exosomes

\section{Background}

Infection of cells by herpesviruses is orchestrated by several viral glycoproteins which function either separately or within multiprotein-complexes to mediate virus entry [1]. Murine gammaherpesvirus 68 (MHV-68) has been extensively used as a model to study the function of gammaherpesvirus glycoproteins [2-5]. Glycoprotein 150 (gp150) of MHV-68 is encoded by open reading frame M7 [6] and has been shown to be virion-associated [7]. It is a positional homolog of gp350/220 of EBV and of gp35/37 of KSHV [6], and it has been shown to be important for virion release [8,9] and antibody evasion [10]. When studying

\footnotetext{
* Correspondence: h.adler@helmholtz-muenchen.de

${ }^{\dagger}$ Equal contributors

${ }^{2}$ Institute of Molecular Immunology, Helmholtz Zentrum München-German

Research Center for Environmental Health, Munich, Germany

Full list of author information is available at the end of the article
}

the function of gp150, both de Lima et al. [9] and Stewart et al. [8] found that mutation of gp150 did not considerably affect lytic replication in vivo and in vitro, except from a moderate deficit in release of extracellular virus. However, Stewart et al. observed a reduction in splenomegaly and in the number of latently infected cells during latency amplification. In contrast, de Lima et al. reported normal latency amplification by gp150-deficient MHV-68. Clearly, to further develop MHV-68 as model for studying the function of gammaherpesvirus glycoproteins in vivo, it is important to know whether gp150 reliably contributes to latency amplification. Here, we present data supporting that gp150 is dispensable for latency amplification but it remains possible that it might play a role under different, so far ill-defined circumstances.

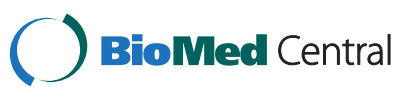




\section{Results and discussion}

Previously, conflicting results have been published on the role of gp150 in latency amplification [8,9], and several hypotheses were raised to explain these discrepancies: i) Stewart et al. introduced a large deletion in the MHV-68 genome to disrupt gp150 which might have inadvertently disrupted additional viral functions, e.g. unknown latency transcripts [2]. On the other hand, the mutations made by de Lima et al. might allow the expression of a truncated but still functional gp150 protein [8]. ii) Stewart et al. investigated only a single mutant while de Lima et al. constructed two independent mutants. The latter can be considered to be a superior strategy to study a gene function [2]. Although Stewart et al. carefully analyzed their mutant by various standard procedures, and even analyzed the viral transcriptome by DNA oligonucleotide microarray, it is formally possible that subtle defects escaped their analysis. iii) Stewart et al. also constructed a BAC-based revertant but BAC-based revertants do not control for problems after virus reconstitution [2].

\section{gp150 and latency amplification}

To address the above mentioned points, we re-evaluated whether gp150 plays a role during latency amplification. For this purpose, we analyzed the following viruses: $i$ ) the original mutant (vgp150 $\Delta$ ) which was constructed in our own laboratory and which has been described in Stewart et al. [8]; ii) a second gp150 mutant (vgp150__2) which we independently reconstituted from the same BAC clone from which the original mutant vgp150 $\Delta$ had been derived - to test for aberrations which might have occurred during reconstitution; iii) a M7-FRT mutant constructed exactly as described by de Lima et al. [9], to test whether the different size and position of the deletion affect the phenotype. All mutant viruses were compared to the revertant virus vgp150R, which had been constructed in our own laboratory and shown to behave exactly like parental (wild-type) virus [8].

To analyze the role of gp150 on latency amplification, we infected C57BL/6 mice intranasally (i.n.) with $5 \times 10^{4}$ PFU. At day 17 after infection, a time point when latency amplification is going on, spleens were harvested and the spleen weights (as a measure of splenomegaly) were determined. Furthermore, the numbers of latently infected cells were determined by a limiting dilution reactivation assay and the latent viral load was measured by quantitative real time PCR. As shown in Figure 1A-C, we did not observe significant differences in any of these parameters (two-tailed Student's $t$-test). To rule out that this finding was specific for C57BL/6 mice, we additionally performed an experiment in BALB/c mice, comparing mutant virus vgp150 $\Delta$ with its revertant (Figure 1D-F). Again, no significant differences were observed (two-tailed Student's $t$-test). Thus, our data suggest that gp150 is not important for latency amplification and clearly show that the different results obtained in the previous studies by Stewart et al. [8] and de Lima et al. [9] are not caused by differences between the mutant viruses which had been used. This finding clearly differs from studies analyzing the role of the MHV- 68 open reading frame M3. M3 is a broad-spectrum chemokine binding protein which inhibits chemokine action $[11,12]$. Disruption of M3 by insertion of a $\beta$-galactosidase expression cassette significantly compromised latency establishment [13], while a M3 mutant generated by insertion of a translational stop codon followed by a frame shift showed normal latency establishment [14]. In this case, the different observations have been attributed to the insertion of the $\beta$-galactosidase expression cassette which may have caused additional adverse effects not related to mutation of M3 [15]. Since the diverse results obtained for gp150 deletions are obviously not related to differences between the mutant viruses which had been used, we speculate that they might be due to differences of the host, the host environment or to subtle differences in the methods applied and time points analyzed. For example, we infected mice at 6 to 8 weeks of age with $5 \times 10^{4} \mathrm{PFU}$, whereas Stewart et al. infected mice at 4 to 6 weeks of age with $4 \times 10^{5}$ PFU [8]. While we analyzed splenomegaly by measuring the spleen weight and the number of ex vivo reactivating cells by ex vivo limiting dilution reactivation assay at day 17 after infection, Stewart et al. used the spleen cell number as a measure of splenomegaly and infectious center assays to determine the number of ex vivo reactivating cells at days 10, 14 and 21 after infection [8]. In addition, different experimental outcomes may correlate with specific properties of the respective mouse facilities. For example, moving Atg16L1-deficient mice from conventional barrier living conditions to an enhanced barrier facility has recently been shown to dramatically change their phenotype [16]. It was shown that this was due to the presence of a particular strain of norovirus in one of the facilities.

\section{gp150 and vaccination}

The MHV-68 model might be very useful to define effective strategies for gammaherpesvirus vaccination [17]. Since it had been proposed that gp350/220 might be a suitable vaccine antigen to protect from EBV-associated diseases [18], the positional homolog of MHV-68, gp150, has been used as a model vaccine in the MHV-68 system. If gp150 contributes to the extent of splenomegaly and to the frequency of latently infected cells during latency amplification in the spleen, as proposed by Stewart et al. [8], it would be reasonable to expect a reduction of both parameters after vaccination with gp150. Indeed, vaccination with a recombinant vaccinia virus expressing gp150 


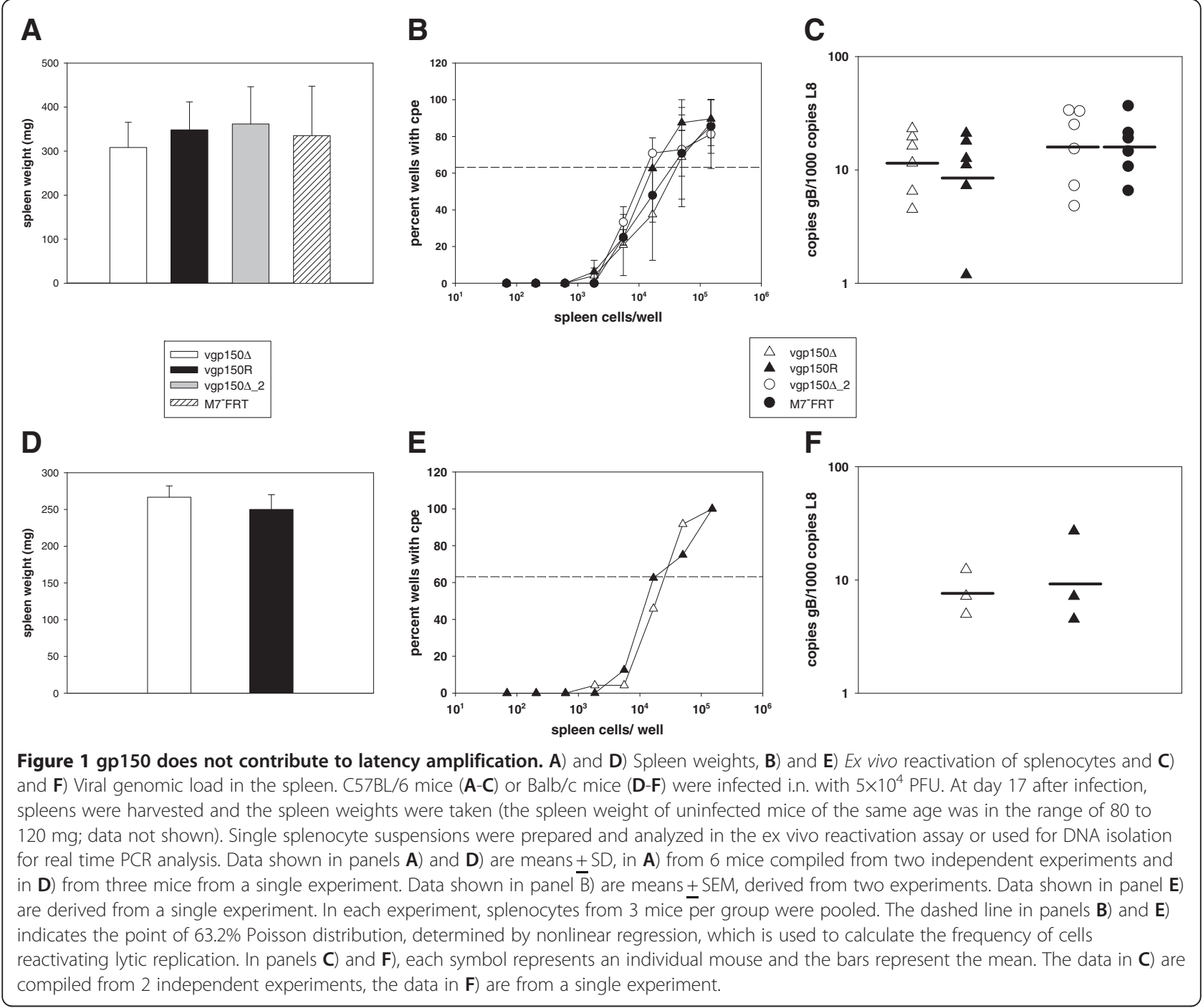

reduced both splenomegaly and the number of latently infected cells in the spleen during latency amplification while it did not reduce lung infection after i.n. MHV-68 challenge infection $[8,19]$. In a separate study, however, an approximately 10 -fold reduction in the lytic virus load in the lungs but no reduction of splenomegaly and of the number of latently infected spleen cells after vaccination with dendritic cells pulsed with a MHC class II-restricted gp150 peptide was observed [20]. Since we did not observe a reduction in splenomegaly and in the number of latently infected cells during latency amplification in the spleen after infection of mice with MHV-68 lacking gp150, we re-evaluated the effect of vaccination with gp150. Specifically, we asked i) whether vaccination of mice with gp150 might induce an anti-gp150 immune response and if so, ii) whether this immune response influences a subsequent MHV-68 challenge infection. For vaccination, we used gp150-containing exosomes. Exosomes are small membrane vesicles which are released into the extracellular compartment during fusion of multivesicular bodies with the plasma membrane and are secreted by various cell types [21]. Exosomes expressing tumor antigens have been shown to be immunogenic, demonstrating the potential of exosomes as vaccines to generate antitumor responses [21,22]. We produced gp150-containing exosomes (293/gp150 exosomes) by transfection of HEK293 cells with an expression plasmid coding for gp150. Exosomes prepared from untransfected HEK293 cells (293 exosomes) served as control. While gp150 was readily detectable by Western Blot in 293/ gp150 exosomes, it was not present in control 293 exosomes (Figure 2A). Consistent with data from Stewart et al. [19], we also detected the gp150 precursor gp130 and alternatively-glycosylated forms of higher molecular weight (Figure 2A). As shown by flow cytometry, 293/ gp150 exosomes bound to beads coated with polyclonal anti-MHV-68 antiserum, demonstrating that gp150 is exposed on the surface of 293/gp150 exosomes 

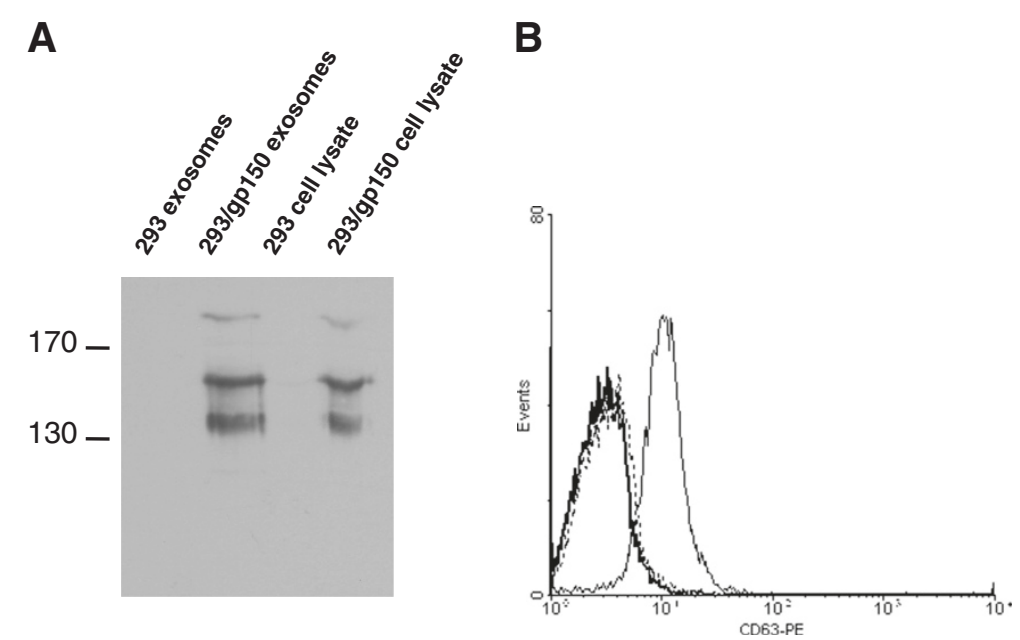

Figure 2 Characterization of exosomes. Exosomes were analyzed for overall gp150 content by Western Blot (panel A) and for gp150 surface content by FACS analysis (panel B). A) Gp150 was detected in purified exosomes and total cell lysates with polyclonal rabbit anti-MHV-68 antibody. Marker sizes (in kD) are indicated on the left. B) Gp150 on the surface of exosomes was detected by binding of exosomes to flow cytometry Protein $\mathrm{G}$ antibody binding beads coated with polyclonal rabbit anti-MHV-68 antibody. Binding of exosomes was visualized by staining for the exosome marker CD63 using a PE-conjugated anti CD63 monoclonal antibody. Solid black line: beads only; dotted black line: beads + 293 exosomes; grey histogram: beads + 293/gp150 exosomes.

(Figure 2B). To test the effect of vaccination with gp150, mice were vaccinated twice (day 0 and 14 ) with $10 \mu \mathrm{g}$ 293/gp150 exosomes or, as a control, with the same amount of 293 exosomes. Nineteen days after the second vaccination (day 33), sera of immunized mice were analyzed by ELISA for the presence of anti-gp150 antibodies, and gp150-specific $\mathrm{T}$ cells were quantified by ELISPOT. Vaccination with 293/gp150 exosomes, but not with control 293 exosomes, induced both humoral (Figure 3A) and cellular gp150-specific immune responses (Figure 3B). The level of the humoral immune response induced by the 293/gp150 exosome vaccination was comparable to that after infection with MHV-68 (Figure 3A). Next, we challenged vaccinated mice by i.n. infection with $5 \times 10^{4}$ PFU of MHV-68 on day 28 and analyzed both lytic and latent infection. Lytic replication was analyzed five days after infection (day 33) by determining lytic virus titers in lung homogenates by standard plaque assay. As shown in Figure 4A, immunization did not influence the amount of lytic virus present in the lungs of infected mice. This result is consistent with previous findings after immunization with a recombinant vaccinia virus expressing gp150 [8,19] but different to findings after vaccination with dendritic cells pulsed with a MHC class II-restricted gp150 peptide [20]. We also analyzed splenomegaly and the number of latently infected cells in the spleen seventeen days after infection (day 45). We found no evidence for an influence of vaccination with gp150 on splenomegaly (Figure 4B), on the number of reactivating cells as determined by limiting dilution reactivation assay (Figure $4 \mathrm{C}$ ) and on the latent viral load as measured by quantitative real time PCR (Figure 4D). While these results are consistent with the data from Woodland et al. [20], they deviate from findings reported by Stewart et al. [19]. The latter might be due to differences of several parameters between the study by Stewart and our study: i) different immunization routes and schedules (subcutaneous vaccination with boost after 28 days vs. intraperitoneal (i.p.) vaccination with boost after 14 days); ii) age and strain of mice (4 week old $\mathrm{BALB} / \mathrm{c}$ mice vs. $6-8$ week old $\mathrm{C} 57 \mathrm{BL} / 6$ mice); iii) time and dose of challenge infection (challenge with $4 \times 10^{5}$ PFU 28 days after second vaccination vs. challenge with $5 \times 10^{4}$ PFU 14 days after second vaccination) and iv) methods and time points used for read-out (virus neutralisation assay, spleen cell number and infectious center assay at days 10, 14, 21 and 28 after challenge infection vs. ELISA, ELISPOT, spleen weight and ex vivo limiting dilution reactivation assay at day 17 after challenge infection). Most importantly, while we used gp150-containing exosomes for vaccination, Stewart et al. used recombinant vaccinia virus expressing gp150. Recombinant vaccinia viruses expressing foreign antigens are powerful vaccines [23]. Yet, vaccination with gp150-containing exosomes also induced a considerable humoral and cellular immune response but did not influence a subsequent MHV-68 challenge infection. This is consistent with more recent findings by Gillet et al. [10], proposing that gp150 is not a significant neutralization target but rather acts as an immunogenic decoy.

\section{Conclusions}

Taken together, our data suggest that gp150 is dispensable for latency amplification. Vaccination with gp150containing exosomes did not affect a subsequent 


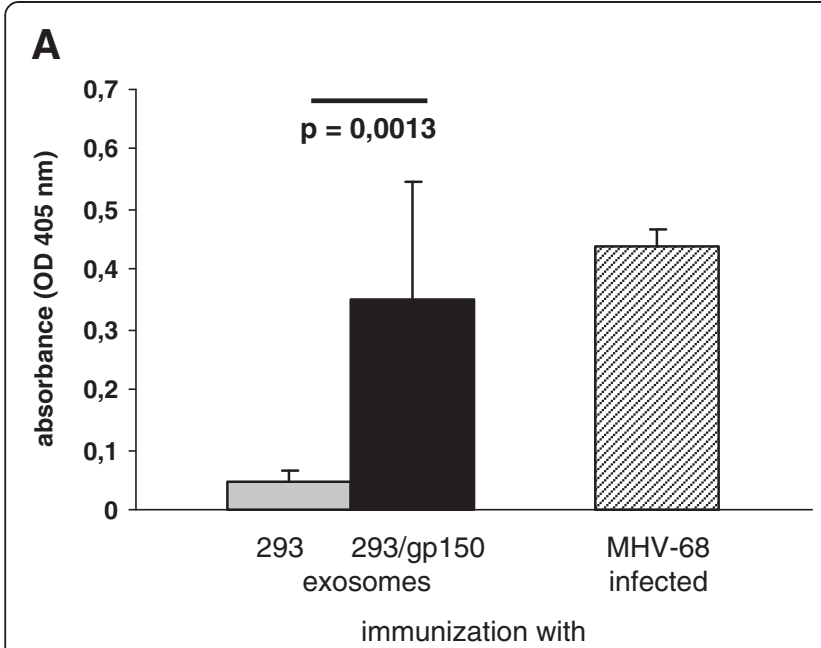

B

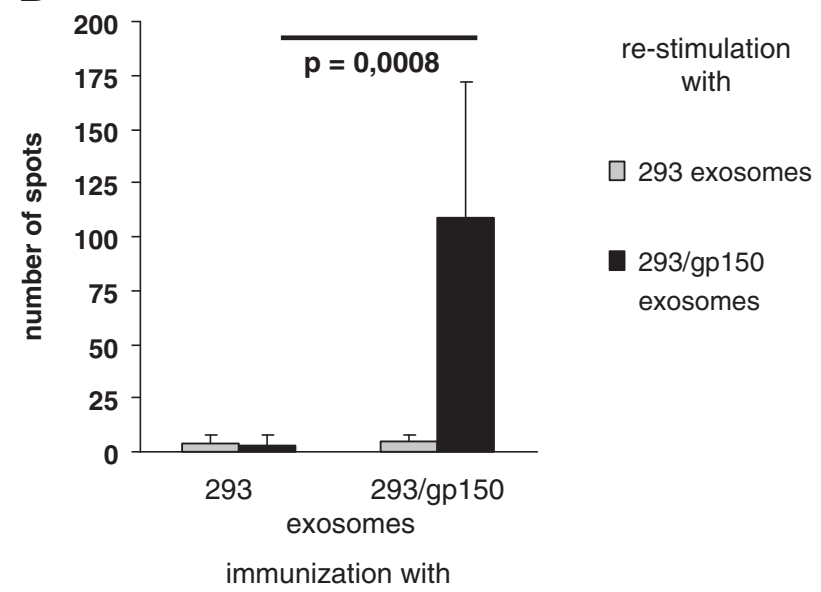

Figure 3 Immune response after vaccination with gp150-containing exosomes. Mice were vaccinated twice (day 0 and 14) with gp150containing exosomes (293/gp150) or as a control, with exosomes prepared from untransfected 293 cells (293). Nineteen days after the second vaccination (day 33), sera were analyzed by ELISA for the presence of anti-gp150 antibodies (panel A). For comparison, antibody levels of MHV-68 infected mice ( $>2$ weeks after infection) are shown. The presence of gp150-specific T-cells was detected by re-stimulation of splenocytes with exosomes (+/-gp150) and subsequent quantification of activated T-cells by IFN- $\gamma$-ELISPOT (panel B). Data shown are means + SD derived from eight (293 exosomes), seven (293/gp150 exosomes) and two (MHV-68 infected) mice, respectively. The Student's t-test (unpaired) was used for statistical analysis.

MHV-68 challenge infection, even though it induced a strong humoral and cellular immune response.

\section{Methods}

\section{Cell lines and virus stocks}

BHK-21 cells (ATCC CCL-10) were grown in GlasgowMEM (PAN Biotech, Aidenbach, Germany) supplemented with $5 \%$ fetal calf serum (FCS), 5\% tryptose phosphate broth, $2 \mathrm{mM}$ L-glutamine, $100 \mathrm{U} / \mathrm{mL}$ penicillin and $100 \mu \mathrm{g} / \mathrm{mL}$ streptomycin. NIH3T3 cells (ATCC CRL1658) and HEK293 cells (ATCC CRL-1573) were grown in DMEM (Invitrogen, Darmstadt, Germany) supplemented with $10 \%$ FCS, $2 \mathrm{mM} \mathrm{L}$-glutamine, $100 \mathrm{U} / \mathrm{mL}$ penicillin and $100 \mu \mathrm{g} / \mathrm{mL}$ streptomycin. Working stocks of viruses were prepared as previously described [24]. Briefly, stocks were grown by infecting BHK-21 cells. After showing complete cytopathic effect (CPE), BHK-21 cells were harvested and the supernatant was used as working stock after two times freezing/thawing the cells and removing cell debris by centrifugation. Virus titers were determined by plaque assays. Briefly, 10 -fold dilutions were incubated on BHK-21 cells for 90 min at $37^{\circ} \mathrm{C}$. After removing the inoculum, cells were incubated for 5 days at $37{ }^{\circ} \mathrm{C}$ with fresh medium containing methylcellulose. Cells were stained with $0.1 \%$ crystal violet solution to determine the number of plaques.

\section{In vivo experiments}

Six to eight weeks old C57BL/6 or BALB/c mice were purchased from Charles River Laboratories (Sulzfeld,
Germany) and housed in individually ventilated cages (IVC) during the MHV-68 infection period. Mice were infected intranasally (i.n.) with $5 \times 10^{4}$ plaque forming units (PFU) of MHV-68. Prior to i.n. infection, mice were anesthetized with ketamine and xylazine. To determine lytic virus titers, the lungs were harvested and homogenized using the FASTPREP ${ }^{\circledR}-24$ instrument (MP Biomedicals, Heidelberg, Germany). After two times freezing and thawing the homogenates, plaque assays were performed with 10-fold dilutions as described above. To determine the frequency of cells carrying virus reactivating from latency, spleens were harvested, single splenocyte suspensions were prepared and analyzed in an ex vivo limiting dilution reactivation assay as described [25]. Briefly, serial threefold dilutions of infected mouse splenocytes were plated on monolayers of $1 \times 10^{4}$ low-passage NIH3T3 cells per well in 96-well tissue culture plates. 24 wells were plated per dilution (starting with $1,5 \times 10^{5}$ cells/well). NIH3T3 cells were screened microscopically for a viral cytopathic effect (cpe) for up to 3 weeks. To differentiate between latently infected cells and infectious virus in the samples, serial threefold dilutions of spleen cells were plated before or after mechanical disruption of viable cells (by two freeze-thaw cycles). No infectious virus was detected in samples of mechanically disrupted cells (data not shown). Frequencies of reactivating cells were calculated on the basis of the Poisson distribution by determining the cell number at which $63.2 \%$ of the wells scored positive for CPE. All animal experiments were in compliance 


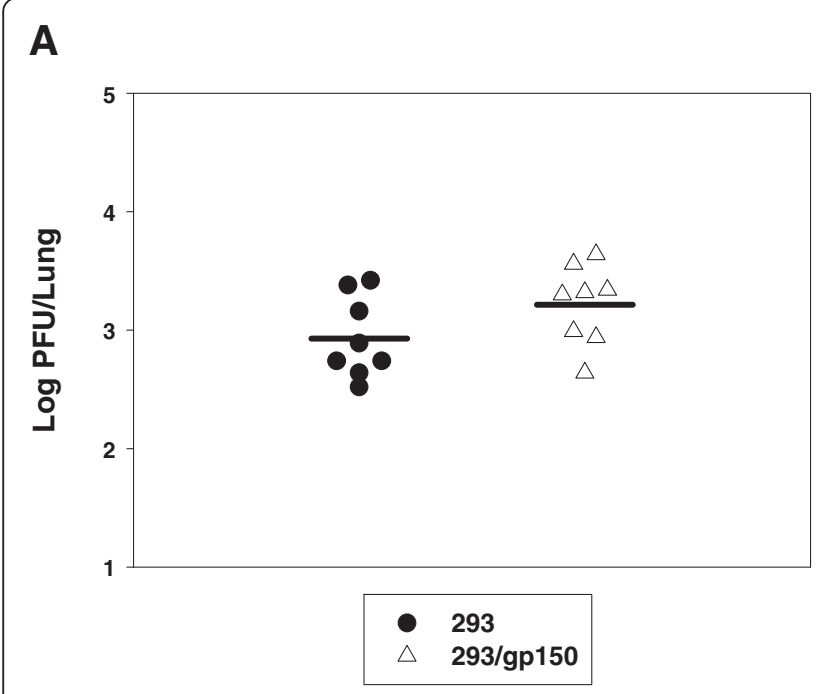

C

\section{B}
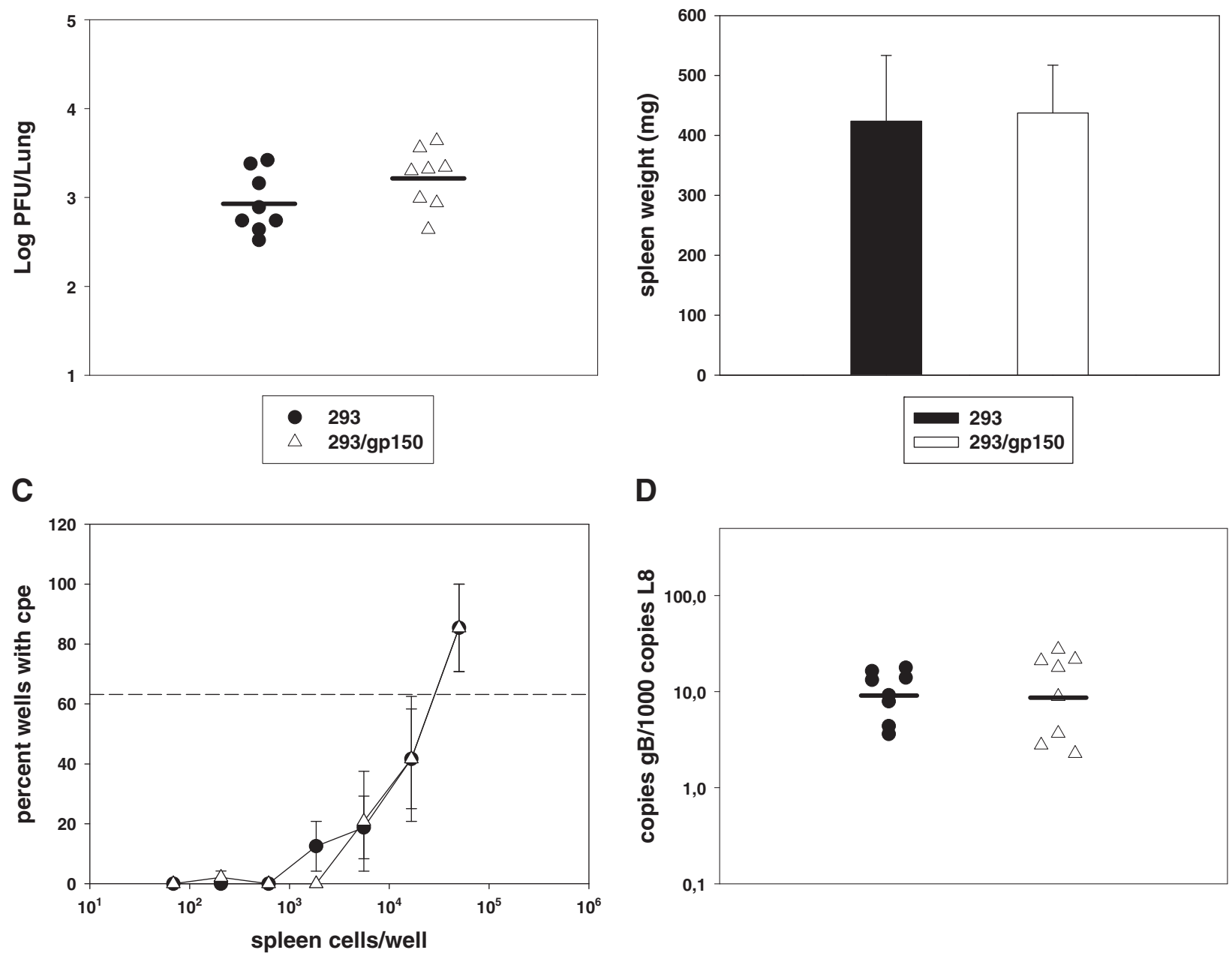

D

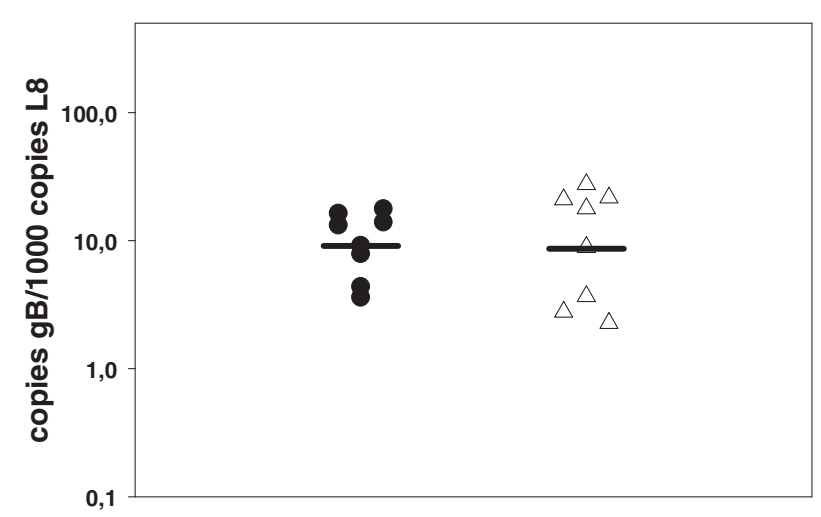

Figure 4 Vaccination with gp150-containing exosomes does not affect a subsequent MHV-68 challenge infection. A) Virus titers in the lung, B) Spleen weights, C) Ex vivo reactivation of splenocytes and D) Viral genomic load in the spleen. Mice were vaccinated twice (day 0 and 14) with gp150-containing exosomes (293/gp150) or as a control, with exosomes prepared from untransfected 293 cells (293). On day 28, mice were challenged by i.n. infection with $5 \times 10^{4} \mathrm{PFU}$. A) Lytic replication was analyzed five days after infection (day 33 ) by determining virus titers in lung homogenates by standard plaque assay. Each symbol represents an individual mouse and the bars represent the mean. The data are compiled from 2 independent experiments. B) to D) Seventeen days after infection (day 45), spleens were harvested and the spleen weights were taken (panel B). Single splenocyte suspensions were prepared and analyzed in the ex vivo reactivation assay (panel C) or used for DNA isolation for real time PCR analysis (panel D). Data shown in panel B) are means + SD from 8 mice compiled from two independent experiments. Data shown in panel $\mathbf{C}$ are means + SEM compiled from two independent experiments. In each experiment, splenocytes from 5 and 3 mice per group, respectively, were pooled. The dashed line in panel $\mathbf{C}$ ) indicates the point of 63.2\% Poisson distribution, determined by nonlinear regression, which is used to calculate the frequency of cells reactivating lytic replication. In panel $\mathbf{D}$ ), each symbol represents an individual mouse and the bars represent the mean. The data are compiled from 2 independent experiments. There were no significant differences in virus titers in the lung (panel A), spleen weights (panel B), ex vivo reactivation of splenocytes (panel C) and viral genomic load in the spleen (panel D). The two-tailed Student's t-test was used for statistical analysis.

with protocols approved by the local Animal Care and Use Committee (District Government of Upper Bavaria; permit number 124/08).

\section{Measurement of latent viral load by quantitative real time PCR}

Viral load in the spleens of infected mice was determined by quantitative real-time PCR using the ABI 7300 Real
Time PCR System (Applied Biosystems, Foster City, CA) as described [26]. Briefly, DNA was extracted from spleen cells using the QIAmp DNA Mini Kit (Qiagen, Hilden, Germany) and quantified by UV spectrophotometry. Amplification of $100 \mathrm{ng}$ of DNA per reaction was performed with Taqman universal PCR master mix and universal cycling conditions (Applied Biosystems, Foster City, CA). Using primers and probes as described [27], a $70 \mathrm{bp}$ 
region of the MHV-68 glycoprotein B (gB) gene was amplified and viral DNA copy numbers were quantified. A standard curve was created using known amounts of a plasmid containing the HindIII-N fragment of MHV68 encompassing the gB gene. The murine ribosomal protein L8 (rpl8) was amplified in parallel and used to normalize for input DNA between samples. The primer and probe sequences for L8 were as follows: Forward: 5'-CATCCCTTTGGAGGTGGTA-3'; Reverse: 5'CATCTCTTCGGATGGTGGA-3' and Probe: 5'-ACCACCAGCACATTGGCAAACC-3'. A standard curve for rpl8 was generated by serial 10 fold dilution of a plasmid containing rpl8 (RZPD clone IRAVp968B01123D6, RZPD, Berlin, Germany). The data are presented as viral genome copy numbers relative to the copy number of L8. The quantification limit was set at 50 copies per sample, according to published recommendations [28].

\section{Production and characterization of gp150-carrying exosomes}

To generate gp150-carrying exosomes, 293 cells were transfected with an expression plasmid coding for gp150, and three days later, exosomes were prepared from the supernatant. Control exosomes were prepared in the same way from untransfected 293 cells. Briefly, exosomes were subjected to sequential centrifugation steps $(300 \mathrm{~g}$ for $10 \mathrm{~min}, 5000 \mathrm{~g}$ for $10 \mathrm{~min}$ and $100.000 \mathrm{~g}$ for $120 \mathrm{~min}$ ). The pelleted exosomes were washed and resuspended in PBS containing proteinase inhibitors (Complete Mini Proteinase Inhibitor Cocktail, Roche, Penzberg, Germany). The protein content was analyzed in a BCA protein microassay (Pierce, Rockford, USA). Exosomes were analyzed for overall gp150 content by Western Blot and for gp150 surface content by FACS analysis. For Western Blotting, $30 \mu \mathrm{g}$ of exosomes were lysed in sample buffer, separated by SDS-PAGE and transferred onto a HybondECL membrane (GE Healthcare, Munich, Germany). gp150 was detected by incubating the membrane with polyclonal rabbit anti-MHV-68 antibody [29], followed by peroxidase-conjugated donkey anti-rabbit antibody (Jackson Laboratories, Newmarket, UK). Blots were developed using ECL reagent (GE Healthcare, Munich, Germany) according to the instructions of the manufacturer. For FACS analysis, flow cytometry Protein G antibody binding beads (Polysciences Europe GmbH, Eppelheim, Germany) were coated with polyclonal rabbit anti-MHV68 antibody for $30 \mathrm{~min}$ at room temperature. After washing with PBS and blocking with RPMI/10\% FCS, beads were incubated with exosomes for two hours at room temperature. After washing, bound exosomes were detected by incubation with PE-conjugated anti CD63 monoclonal antibody (Immunotools, Friesoythe, Germany). CD63, a member of the tetraspanin family, is a marker protein for exosomes [21].

\section{Vaccination with exosomes and challenge infection}

Mice were vaccinated twice (day 0 and 14) with gp150containing exosomes (293/gp150 exosomes) or as a control, with exosomes prepared from untransfected 293 cells (293 exosomes). Vaccination was performed i.p. with a mixture of $10 \mu \mathrm{g}$ of exosomes and $10 \mathrm{nmol} \mathrm{CpG-}$ ODN 1668 (InvivoGen, San Diego, USA) in a total volume of $200 \mu \mathrm{l}$ PBS per mouse. Fourteen days after the second vaccination (day 28), mice were challenged with $5 \times 10^{4}$ PFU of MHV- 68 .

\section{ELISPOT assay for determination of gp150-specific T cells}

To determine gp150-specific T-cells, splenocytes from vaccinated mice were plated onto precoated ELISPOT plates in a densitiy of $10^{6}$ cells/well and restimulated in vitro with $10 \mu \mathrm{g}$ of exosomes (+/- gp150). After $24 \mathrm{~h}$, activated T-cells were quantified using a mouse IFN- $\gamma$ ELISPOT Kit (eBioscience, Frankfurt, Germany) as recommended by the manufacturer, and spots were counted by inspection under a dissecting microscope.

\section{ELISA for determination of gp150-specific antibodies}

To determine gp150-specific antibodies in sera of vaccinated mice, Nunc Maxisorp plates (Nunc, Wiesbaden, Germany) were coated overnight with $3 \mu \mathrm{g} / \mathrm{ml}$ of MHV68 lysate. The lysate was prepared by disruption of MHV-68 via dilution in PBS with $0.05 \%$ Triton X-100 as described [30]. After washing with PBS/Tween, plates were blocked with RPMI containing 10\% FCS for 1 hour and washed again. Then, fifty-fold serum dilutions were incubated for two hours. After washing, bound antibody was detected with HRP-conjugated rat anti-mouse antibody (Promega, Mannheim, Germany) using TMB (Becton Dickinson, Heidelberg, Germany) as substrate and, after stopping with $1 \mathrm{M}$ phosphoric acid, reading the absorbancy at $450 \mathrm{~nm}$.

\section{Statistical methods}

If not otherwise indicated, data were analyzed by twotailed Student's $t$-test.

\section{Competing interests}

The authors declare that they have no competing interests.

\section{Authors' contributions}

$\mathrm{RZ}$ and $\mathrm{HA}$ conceived and designed the experiments. RR, SO, BS and HA performed the experiments. RR, SO, RZ and HA analyzed the data. HA wrote the paper. All authors read and approved the final manuscript.

\section{Acknowledgements}

This work was supported by grants from the BMBF (NGFNplus, FKZ PIM01GS0802-3) and the Wilhelm Sander-Stiftung (grant 2009.046.1) to H.A., from the Japan Herpesvirus Infections Forum to S.O., and from the Deutsche Krebshilfe (grant 107793) to R.Z.. We are grateful to the members of our animal facilities for expert technical assistance. We also thank the members of our group for helpful discussions and B. Adler for critically reading of the manuscript. 


\section{Author details}

${ }^{1}$ Research Unit Gene Vectors, Helmholtz Zentrum München-German Research Center for Environmental Health, Munich, Germany. ${ }^{2}$ Institute of Molecular Immunology, Helmholtz Zentrum München-German Research Center for Environmental Health, Munich, Germany. ${ }^{3}$ Department of Otorhinolaryngology, Klinikum der Universität München, Munich, Germany. ${ }^{4}$ Present address: Department of Virology, Faculty of Medicine, Kyushu University, Fukuoka 812-8582, Japan.

Received: 18 January 2012 Accepted: 3 June 2012

Published: 9 June 2012

\section{References}

1. Spear PG, Longnecker R: Herpesvirus entry: an update. J Virol 2003 77:10179-10185.

2. Gillet $L$, Stevenson PG: Evidence for a multiprotein gamma-2 herpesvirus entry complex. J Virol 2007, 81:13082-13091.

3. Gillet L, Colaco S, Stevenson PG: Glycoprotein B switches conformation during murid herpesvirus 4 entry. J Gen Virol 2008, 89:1352-1363.

4. Gillet L, Colaco S, Stevenson PG: The murid herpesvirus- $4 \mathrm{gH} / \mathrm{gL}$ binds to glycosaminoglycans. PLoS One 2008, 3:e1669.

5. May JS, Colaco S, Stevenson PG: Glycoprotein $M$ is an essential lytic replication protein of the murine gammaherpesvirus 68. J Virol 2005, 79:3459-3467.

6. Virgin HWIV, Latreille P, Wamsley P, Hallsworth K, Weck KE, Dal Canto AJ, et al: Complete sequence and genomic analysis of murine gammaherpesvirus 68. J Virol 1997, 71:5894-5904.

7. Stewart JP, Janjua NJ, Pepper SDV, Bennion G, Mackett M, Allen T, et al: Identification and characterization of murine gammaherpesvirus $68 \mathrm{gp}$ 150: a virion membrane glycoprotein. J Virol 1996, 70:3528-3535.

8. Stewart JP, Silvia OJ, Atkin IM, Hughes DJ, Ebrahimi B, Adler H: In vivo function of a gammaherpesvirus virion glycoprotein: influence on B-cell infection and mononucleosis. J Virol 2004, 78:10449-10459.

9. de Lima BD, May JS, Stevenson PG: Murine Gammaherpesvirus 68 Lacking gp150 Shows Defective Virion Release but Establishes Normal Latency In Vivo. J Virol 2004, 78:5103-5112.

10. Gillet L, May JS, Colaco S, Stevenson PG: The murine gammaherpesvirus68 gp150 acts as an immunogenic decoy to limit virion neutralization. PLoS One 2007, 2:e705

11. Van Berkel V, Barret J, Tiffany HL, Fremont DH, Murphy PM, McFadden $G$, et al: Identification of a gammaherpesvirus selective chemokine binding protein that inhibits chemokine action. J Virol 2000, 74:6741-6747

12. Parry CM, Simas JP, Smith VP, Stewart CA, Minson AC, Efstathiou S, et al: A broad spectrum secreted chemokine binding protein encoded by a herpesvirus. J Exp Med 2000, 191:573-578.

13. Bridgeman A, Stevenson PG, Simas JP, Efstathiou S: A secreted chemokine binding protein encoded by murine gammaherpesvirus- 68 is necessary for the establishment of a normal latent load. J Exp Med 2001, 194:301-312

14. Van Berkel V, Levine B, Kapadia SB, Goldman JE, Speck SH, Virgin HWIV: Critical role for a high-affinity chemokine-binding protein in gammaherpesvirus-induced lethal meningitis. J Clin Invest 2002, 109:905-914.

15. Barton E, Mandal P, Speck SH: Pathogenesis and Host Control of Gammaherpesviruses: Lessons from the Mouse. Annu Rev Immunol 2011 29:351-397

16. Cadwell K, Patel KK, Maloney NS, Liu TC, Ng AC, Storer CE, et al: Virus-plussusceptibility gene interaction determines Crohn's disease gene Atg16L1 phenotypes in intestine. Cell 2010, 141:1135-1145.

17. Stevenson PG, Efstathiou S: Immune mechanisms in murine gammaherpesvirus-68 infection. Viral Immunol 2005, 18:445-456.

18. Epstein MA, Morgan AJ, Finerty S, Randle BJ, Kirkwood JK: Protection of cottontop tamarins against Epstein-Barr virus-induced malignant lymphoma by a prototype subunit vaccine. Nature 1985, 318:287-289.

19. Stewart JP, Micali N, Usherwood EJ, Bonina L, Nash AA: Murine gammaherpesvirus 68 glycoprotein 150 protects against virus-induced mononucleosis: a model system for gamma-herpesvirus vaccination. Vaccine 1999, 17:152-157.

20. Woodland DL, Usherwood EJ, Liu L, Flano E, Kim IJ, Blackman MA: Vaccination against murine gamma-herpesvirus infection. Viral Immunol 2001, 14:217-226
21. Cho JA, Yeo DJ, Son HY, Kim HW, Jung DS, Ko JK, et al: Exosomes: a new delivery system for tumor antigens in cancer immunotherapy. Int $\rfloor$ Cancer 2005, 114:613-622.

22. Chaput N, Schartz NE, Andre F, Zitvogel L: Exosomes for immunotherapy of cancer. Adv Exp Med Biol 2003, 532:215-221

23. Sutter G, Staib C: Vaccinia vectors as candidate vaccines: the development of modified vaccinia virus Ankara for antigen delivery. Curr Drug Targets Infect Disord 2003, 3:263-271.

24. Adler $H$, Messerle $M$, Wagner $M$, Koszinowski UH: Cloning and mutagenesis of the murine gammaherpesvirus 68 genome as an infectious bacterial artificial chromosome. J Virol 2000, 74:6964-6974.

25. Adler $\mathrm{H}$, Messerle M, Koszinowski UH: Virus reconstituted from infectious bacterial artificial chromosome (BAC)-cloned murine gammaherpesvirus 68 acquires wild-type properties in vivo only after excision of BAC vector sequences. J Virol 2001, 75:5692-5696.

26. Flach B, Steer B, Thakur NN, Haas J, Adler H: The M10 locus of murine gammaherpesvirus 68 contributes to both the lytic and the latent phases of infection. J Virol 2009, 83:8163-8172.

27. Weinberg JB, Lutzke ML, Alfinito R, Rochford R: Mouse strain differences in the chemokine response to acute lung infection with a murine gammaherpesvirus. Viral Immunol 2004, 17:69-77.

28. Vaerman $J L$, Saussoy $P$, Ingargiola I: Evaluation of real-time PCR data. J Biol Regul Homeost Agents 2004, 18:212-214

29. Steer B, Adler B, Jonjic S, Stewart JP, Adler H: A gammaherpesvirus complement regulatory protein promotes initiation of infection by activation of protein kinase Akt/PKB. PloS One 2010, 5:e11672.

30. Stevenson PG, Doherty PC: Kinetic analysis of the specific host response to a murine gammaherpesvirus. J Virol 1998, 72:943-949.

doi:10.1186/1743-422X-9-107

Cite this article as: Ruiss et al:: Murine gammaherpesvirus 68 glycoprotein 150 does not contribute to latency amplification in vivo. Virology Journal 2012 9:107.

\section{Submit your next manuscript to BioMed Central and take full advantage of:}

- Convenient online submission

- Thorough peer review

- No space constraints or color figure charges

- Immediate publication on acceptance

- Inclusion in PubMed, CAS, Scopus and Google Scholar

- Research which is freely available for redistribution
C Biomed Central 